\title{
Effects of Rotation in Low-Metallicity Stars
}

\author{
Sara R. Heap \\ Bethesda MD 20817-6740 \\ Thierry Lanz \\ University of Maryland, College Park MD 20742 \& \\ NASA's Goddard Space Flight Center, Greenbelt MD 20771
}

\begin{abstract}
We have carried out a spectral analysis of 17 O-type stars in the Small Magellanic Cloud (SMC). We find several lines of evidence that rotation plays an important role in massive stars in this low-metallicity galaxy $(\mathrm{Z}=0.2$ $\left.\mathrm{Z}_{\odot}\right)$ : (1) strong enrichment of nitrogen in half the program stars due to rotational mixing of processed material in the core up to the surface; (2) reduction of the effective gravity by the centrifugal force; (3) apparent anomalies in cluster isochrones that imply some stars are rotating at critical velocities.
\end{abstract}

Both theory and observations suggest that rotation is a more important factor in massive stars of low metallicity (Z). Low-Z stars lose less angular momentum due to their weaker winds (Maeder \& Meynet 2001). They may also be born as faster rotators, because they are smaller than their galactic counterparts. Observations support this trend with metallicity in revealing that the fraction of Be stars (rapid rotators) to total $(\mathrm{Be}+\mathrm{B})$ stars in clusters increases toward lower metallicity (Maeder et al. 1999). This makes the SMC, a low metallicity galaxy $\left(\mathrm{Z}=0.2 \mathrm{Z}_{\odot}\right)$, an ideal environment for discerning the effects of rotation.

We have obtained high-resolution spectra of 17 O-type stars in the SMC using STIS on HST, FUSE, and ESO or AAT (c.f. Walborn et al. 2000). We used the new line-blanketed NLTE photospheric model atmospheres of Lanz \& Hubeny (2002) to derive the fundamental properties of each star (Heap et al. 2002). We then compared the observed properties to evolutionary models with/without rotation. We find that the observations strongly favor the rotating models as discussed below.

1. Nitrogen enhancement. We derived preliminary $\mathrm{N}$ abundances for all 17 program stars with results as shown in Figure 1. Three O stars (white star symbols) have low $\mathrm{N}$ abundances typical of $\mathrm{SMC}$ nebulae, $[\mathrm{N}]=6.45$. Such stars must be slow rotators. Nine of 17 program stars (black stars) show a surface enrichment of $\mathrm{N}$ up to a solar level, $[\mathrm{N}]=7.92$ - even higher than predicted by rotating models. The high observed $\mathrm{N}$ abundances suggest that SMC $\mathrm{O}$ stars generally rotate more rapidly than assumed in the models (ZAMS $v_{\text {rot }}=300$ $\mathrm{km} / \mathrm{s}$ ), and/or rotational mixing is more efficient than assumed. 


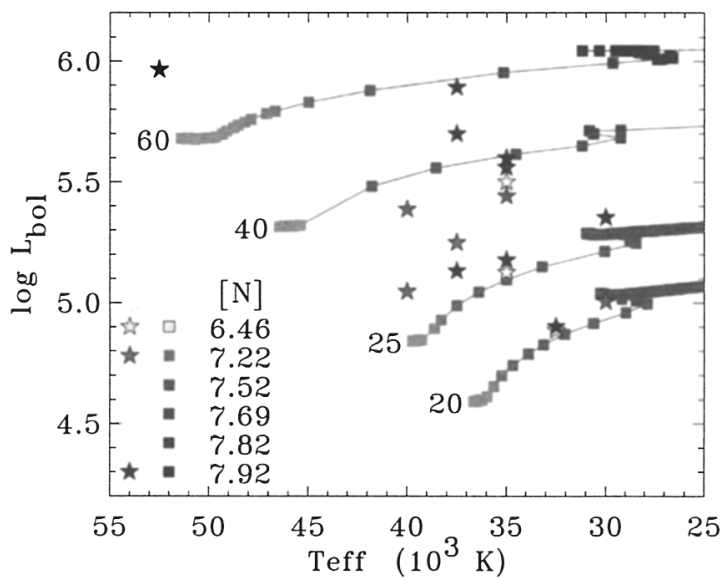

Figure 1. Comparison of observed atmospheric N abundances in SMC Otype stars with theoretical surface $\mathrm{N}$ abundances from the rotating models of Maeder \& Meynet (2001).

2. Mass Discrepancy. Most, but not all, program stars have spectroscopic masses $\left(M_{\mathrm{sp}}\right)$ that are lower than indicated by their position on the HRD. The mass discrepancy is usually worse for stars with a high $\mathrm{N}$ abundance than for stars with their original (low) $\mathrm{N}$ abundance. Rotation can cause this mass discrepancy by decreasing $g_{\text {eff }}$ and therefore $M_{\mathrm{sp}} \propto g_{\mathrm{eff}} R^{2}$ and/or by increasing the luminosity $\left(M_{\mathrm{ev}}\right)$.

3. Anomalous Isochrone. The program stars include 7 members of NGC 346 , a young cluster in the SMC. One of the seven, NGC 346-355 $\left(\mathrm{T}_{\text {eff }} \sim 52,500\right.$ $\mathrm{K}, \log L \sim 6.0)$ stands out as being apparently younger $(t \sim 1 \mathrm{Myr})$ than the others yet showing a high (solar) $\mathrm{N}$ abundance. We suggest that these anomalies can be explained if this star is rotating at its critical velocity. Theoretical studies (Maeder 1987) show that a star rotating at critical velocity will evolve up and to the left on the HRD, mimicking a younger, more massive star. Such a star will be highly enriched in nitrogen. This description nicely fits NGC 346-355.

\section{References}

Heap, S., Lanz, T. et al. 2002, to be submitted to ApJ

Lanz, T. \& Hubeny, I. 2002, astro-ph/0210157

Maeder, A. 1987, A\&A, 178, 159

Maeder, A., Grebel, E., Mermilliod, J.C. 1999, A\&A 346, 459

Maeder, A. \& Meynet, G. 2001, A\&A, 373, 555

Walborn, N., Lennon, D., Heap, S. et al. 2000, PASP, 112, 1243 